\title{
Tagging Firefighter Activities at the Emergency Scene: Summary of AAIA'15 Data Mining Competition at Knowledge Pit
}

\author{
Michał Meina, Andrzej Janusz, \\ Krzysztof Rykaczewski \\ Institute of Mathematics \\ University of Warsaw \\ Warsaw, Poland \\ \{mich,janusza,krykaczewski\}@ mimuw.edu.pl
}

\author{
Dominik Ślęzak \\ Institute of Mathematics \\ University of Warsaw \\ and \\ Infobright Inc. \\ Warsaw, Poland \\ slezak@mimuw.edu.pl
}

\author{
Bartosz Celmer and Adam Krasuski \\ Section of Computer Science \\ The Main School of Fire Service \\ Warsaw, Poland \\ bart.celmer@gmail.com \\ krasuski@inf.sgsp.edu.pl
}

\begin{abstract}
In this paper, we summarize AAIA'15 data mining competition: Tagging Firefighter Activities at a Fire Scene, which was held between March 9 and July 6, 2015. We describe the scope and background of the competition. We also reveal details regarding the data set used in the competition, which was collected and tagged specifically for the purpose of this data challenge. We explain the data acquisition process which involved using a body sensor network system consisting of several inertial measurement units and a physiological data sensor. Finally, we briefly discuss submitted results with respect to their possible real-life application in our decision support system.
\end{abstract}

\section{INTRODUCTION}

The emergency scene is considered to be one of the most dangerous and stressful working environments [3]. Each year the numbers of firefighters die or are injured during the fire \& rescue operations. There are many contributing factors which lead to the unsafe events. Those factors are investigated by special commissions and according to [10] $25 \%$ of accidents are caused by bad decision making, $29 \%$ by bad situational awareness and $10 \%$ by bad communication.

The key aspect (regardless of the type of the Incident Management System) comes down to modelling of perception and evaluation of the emergency scene by the Incident Commander (IC) [5], [9], [6]. The relevant incident assessment methodology increases the safety of the rescuers and the chances for success. Information plays a pivotal role in the perception and evaluation. Therefore, there is a strong demand on increasing the sensor density in order to provide more information to the IC. The information reported to the IC must satisfy the information triangle rule [5]. It means that it should be relevant, accurate and timely. All of those aspects - in dynamically changing environment such as a fire incident is - is difficult to satisfy.

The research was partially supported by the Polish National Science Centre - grant 2012/05/B/ST6/03215 and by the Polish National Centre for Research and Development (NCBiR) - grant No. O ROB/0010/03/001 in the frame of Defence and Security Programmes and Projects: "Modern engineering tools for decision support for commanders of the State Fire Service of Poland during Fire\&Rescue operations in the buildings".
During emergency firefighters are mostly concentrated on their principal activities. For the safety reasons they are not able to share in real-time with the IC the information about the current conditions. Paradoxically, as described above, activities currently performed by the firefighters, their physical condition as well as temporal and spatial relation to the fire are crucial for the IC in order to ensure safety operating of the firefighters.

Since the information at the emergency scene is crucial for the operating safety and the personal real-time reporting from firefighters are hampered, the new ways of indirect communication and information sharing are needed. The computerbased systems for human activity recognition may help to reduce the unsafe events, improving the communication and increasing the efficacy of the incident management. Moreover, due to the dangerous environment for human, it could be expected that in the future more and more robot will be involved in operating at the emergency scene. The human activity recognition system may create a bridge for human robot cooperation at the scene.

Human activity recognition using Body Sensor Networks (BSN) is a non-invasive system that is able to deliver information about person locomotion patterns, current posture and specific action performed. In the system that is considered in this paper, a network of inertial measurement units are used. They are able to gather kinematic (motion) data from different parts of the body. This information is then processed using classification techniques [16] (by the body-worn computation unit) in order to estimate activity and passed via radio link to the IC. In such systems like described training data needs to be prepared beforehand and classification procedure needs to take into account power consumption on mobile processing unit and low-radio link throughput.

This paper describes a data mining competition which was organized at the Knowledge Pit platform [14]. Special dataset collected during training exercises of firefighters cadets using custom-built BSN was prepared and made available publicly to encourage a research community to work on new methods and classification models in this particular application. The competition was a part of a broader research addressing a sensory data acquisition at the incident place. The submitted 
results forms very valuable input into the field and forms a comparable platform for future research.

The paper starts from a detailed discussion of the data acquisition process, including our hardware setup, collection of the data and the process of tagging. The third section describes the preprocessing of the data set for the competition and the evaluation scenario of solutions submitted by competitors. This section also includes a brief summary of the competition results. The paper ends with concluding remarks and a draft of our plans for future work.

\section{DATA ACQUISITION}

This section describes data acquisition part of the competition. In the first paragraph we will discuss our hardware design and processing challenges connected with on-line processing the data. Second paragraph will summarize recording sessions and the final paragraph will cover process of annotating the data set.

\section{A. Hardware setup}

The data acquisition hardware is consisted of sensors (seven IMUs and physiological data sensor) and Data Acquisition Unit (DAU). In order to overcome clock synchronization issues all IMUs are connected via physical link to real-time embedded system that streams the data to DAU. Physiological sensor has it own clock synchronization system, therefore information can be streamed via Bluetooth. Moreover, DAU provides simple web interface (accessible via Wi-Fi connection) to control recording trials (starting, stopping and monitoring data collection process).

In our setup we use:

- $7 \times$ IMU (Polulu AltIMU-9 rev-4) - 3-axis accelerometer ( $\pm 16 \mathrm{~g}$ dynamic range) and gyroscope $\left( \pm 2000^{\circ} / s\right.$ maximum angular rate) with 16 bit signed integer resolution, two IMUs for legs, two for hands and arms and one for back,

- Real-time system embedded — based on Arduino Micro prototype platform, connected via USB interface to DAU,

- Data Acquisition Unit - based on Odroid-U3+ with external battery, additional Bluetooth and Wi-Fi module,

- Physiological data sensor - Equivital EQ02 SEM,

- $\quad$ Communication nodes - XBee-PRO ${ }^{\circledR} 868$.

In a low level point of view, IMU are two different sensors, one for measuring accelerations one for angular rates. Both, accelerometer and gyroscope provide 16 bit signed integer for each axis (horizontal, vertical and altitudinal), and communicate via $I^{2} C$ bus. In order to provide the data with zero-time offset between different IMUs, we used real time system (microcontroller) and we have prepared parallel communication version of $I^{2} C$ library. The library enables us to read the sensor readings from all IMUs at the same time with 1000 samples per second. We decided, however, to gather the data with the frequency about $200 \mathrm{~Hz}$ which is a sufficient for classification

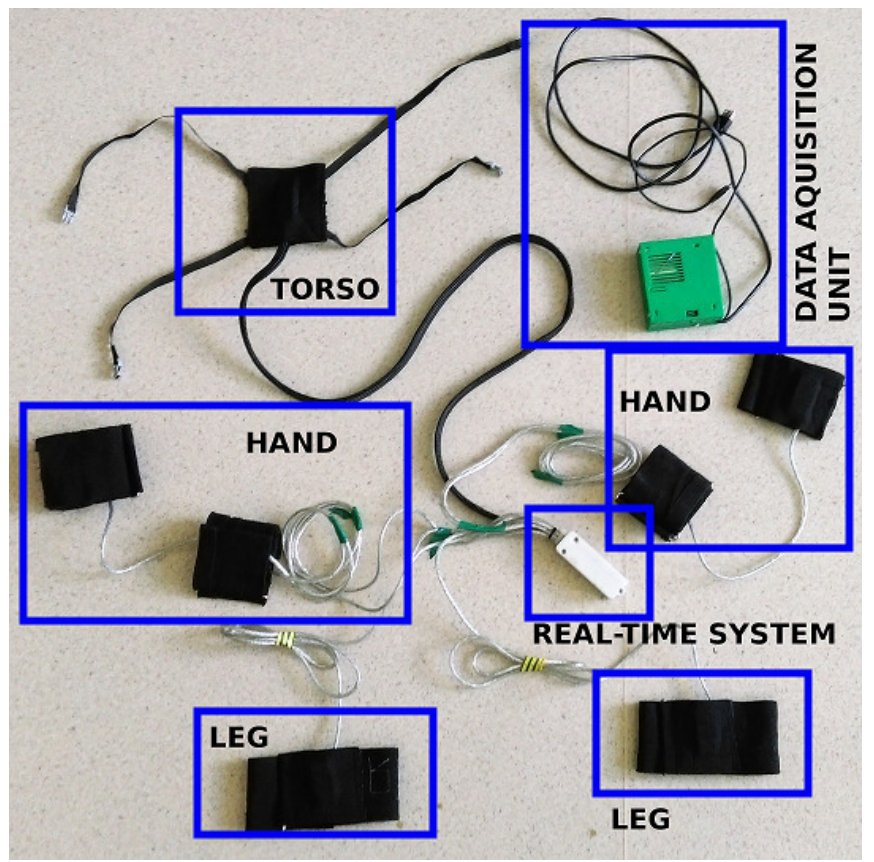

Fig. 1. Hardware setup that is able to gather motion data from different parts of the body. IMUs are kept in a custom $3 \mathrm{D}$ printed casing and mounted to the body using elastic bands.

purposes, while providing low noise and enough accuracy for the most of the sudden movements.

Physiological data (ECG waveform, heart rate, breathing rate and skin temperature) was recorded by Equivital ${ }^{1}$ on internal storage and redundantly sent via Bluetooth to DAU.

During recording session the raw data was stored on DAU using binary format. Single sample, consist of readings from all sensor which sums up to 680 bits (16 bit $\cdot 2 \cdot 7$ sensors . 3 axis +8 bit time stamp). In the real application, however, the minimum required bandwidth for the data transmission is higher because of network maintaining routines overhead, so final minimal bandwidth was more than $49.8 \mathrm{kB} / \mathrm{s}$ for each subject. Reliable transmission of the raw data through radio for longer distances $(1-2 \mathrm{~km})$ while maintaining reasonable power consumption is very challenging or almost impossible task. LTE or Wi-Fi connections enables us to stream such amount of data, but while using these technologies problems with the power consumption arise (see [13], [7]). Although low frequency radios have low bandwidth, the power consumption is sufficient - this setup, however, imposes the need of processing the data locally and transmission partially preprocessed data.

Most intuitive and robust design is that the data is processed on DAU and only the classification result should are sent to remote server. On the other hand there is no implementation of more sophisticated classification system dedicated to work on embedded system (due to floating point precision and lack of processing power). Therefore, we have used Odroid-U3+ platform based on quad-core Cortex-A9. This platform has a two scaling frequencies options and possibility of choosing the

${ }^{1}$ http://www.equivital.co.uk/ 
number of cores being used. This setup gives us ability to scale power consumption depending on classification algorithm.

\section{B. Training session recording}

Recording sessions took place from January till February 2015 at the Main School of Fire Service in Warsaw. Sixteen cadets were recorded during a training session of fire incident. There were two scenarios of the trial and each of them was repeated three times. However, we did not use breathing apparatus and real-life obstacles which changes significantly kinematics of a subject in real incidents.

Recording procedure was as follows: before session each sensor was calibrated on-place and checked for errors using gravitational force measurements in different sensor orientations. Two cadets participated in each session, whereas only one of them was wearing the sensors. Second cadet was later responsible for maintaining equipment on field. Those preparations lasted for 30 minutes. Each session was recorder using two cameras. Quality of the kinematic recordings was monitored with respect to throughput of the data using mobile device. At the beginning of each trial subject was asked to jump for three times to estimate precise time shift between video and kinematic data. Cadet performed two significantly different scenarios in three trials (each of them lasted 3-5 minutes), after that wearable sensor was transferred to the second cadet. At the end of the trial, the sensor on-body mounting was checked for displacement and the data was archived.

Recording of two cadets usually lasts for three hours and it requires commitment of three additional persons. After manual examination and verification of data quality we have chosen only eight out of fifteen sets of recordings. In the most of cases the reason for the elimination of a recording was either a sensor malfunction or a displacement of the sensor during the recorded training session.

\section{Tagging the data set}

Tagging was prepared using video material on which acceleration from torso sensor was overlaid with precise time synchronization using marker (three jumps that was very easily observable in sensory data). Time series was divided into episodes of actions and there was always left a small gap between episodes in order not to introduce noisy transition phases (for example, deceleration from running to walking).

We decided to prepare two set of labels (tags), namely:

- posture which included: crawling, crouching, jumping, movement, standing, stooping,

- activity - few tags specifying short and specific actions. It included: carrying, carrying_hammer, carrying_nozzle, carrying_hose, hammer_striking, hose_throwing, ladder_down, ladder_up, manipulating, mounting_hose, nozzle_usage, running, searching, signal_hose_pullback, signal_water_first, signal_water_main, stairs_down_fast, stairs_up, stairs_up_fast, standing_up, starting, step, stopping, taking_equipment, taking_hammer, waiting, walking.
TABLE I. OCCURRENCES OF POSTURE TAG IN FINAL DATA SET.

\begin{tabular}{|c|c|c|}
\hline Posture & Number of occurrences & Total time [s] \\
\hline \hline crawling & 27 & 461.75 \\
\hline crouching & 125 & 1109.0 \\
\hline jumping & 96 & 242.75 \\
\hline movement & 613 & 3083.0 \\
\hline standing & 423 & 1524.25 \\
\hline stooping & 267 & 645.0 \\
\hline
\end{tabular}

TABLE II. MOS POPULAR TAGS IN SECOND GROUP.

\begin{tabular}{|c|c|c|}
\hline Activity & Number of occurrences & Total time [s] \\
\hline \hline ladder_down & 24 & 230.5 \\
\hline ladder_up & 23 & 237.5 \\
\hline manipulating & 482 & 1787.75 \\
\hline no_action & 51 & 122.5 \\
\hline nozzle_usage & 48 & 715.5 \\
\hline running & 337 & 1800.25 \\
\hline searching & 25 & 439.0 \\
\hline signal_hose_pullback & 2 & 4.25 \\
\hline signal_water_first & 33 & 63.0 \\
\hline signal_water_main & 30 & 51.25 \\
\hline signal_water_stop & 4 & 9.75 \\
\hline stairs_down & 32 & 175.0 \\
\hline stairs_up & 69 & 294.0 \\
\hline starting & 49 & 119.5 \\
\hline stopping & 47 & 123.25 \\
\hline striking & 68 & 339.75 \\
\hline taking_hammer & 47 & 68.75 \\
\hline throwing_hose & 84 & 208.5 \\
\hline walking & 94 & 273.25 \\
\hline
\end{tabular}

However, not all of these tags were fully independent. For example, carrying always occurs after one of carrying_hammer, carrying_nozzle, carrying_hose. Therefore, not all combinations exist.

First tag in the activity labels specifies main action and other introduce detailed activities. The idea behind this was to show hierarchy of events.

\section{The acquired collection of data}

The data set consist of 16 recording session (one for each cadet), while only 8 is most suitable for analysis. Each session is composed from kinematic data divided into six trials (three trials for two scenarios) accelerometer and gyroscope in three axes from seven mounting points described before (see Fig. 2 as example of such session). There are 3065664 records as a whole summing up to $4.25 \mathrm{~h}$ of data.

TABLE III. EIGHTEEN MOST POPULAR COMBINATION OF TAGS.

\begin{tabular}{|c|c|c|}
\hline Posture & Activities & Total time [s] \\
\hline \hline movement & running & 727.25 \\
\hline movement & running carrying_hose carrying & 537.50 \\
\hline crawling & searching & 439.00 \\
\hline movement & running carrying_hammer carrying & 436.00 \\
\hline crouching & nozzle_usage & 432.25 \\
\hline crouching & manipulating & 389.00 \\
\hline stooping & manipulating & 350.00 \\
\hline standing & striking & 339.75 \\
\hline crouching & manipulating mounting_hose & 261.75 \\
\hline standing & nozzle_usage & 260.00 \\
\hline standing & manipulating carrying_nozzle carrying & 252.50 \\
\hline standing & manipulating & 248.50 \\
\hline movement & ladder_up carrying_nozzle carrying & 237.50 \\
\hline movement & ladder_down carrying_nozzle carrying & 197.75 \\
\hline movement & stairs_down & 168.50 \\
\hline stooping & throwing_hose & 158.75 \\
\hline movement & stairs_up carrying_nozzle carrying & 137.25 \\
\hline movement & stairs_up carrying_hammer carrying & 132.75 \\
\hline
\end{tabular}





Fig. 3. MDS embedding for selected activities (colours denotes different test subjects). Notice that in the last figure dots denotes stairs_down tag, whereas crosses stairs_up.

Physiological data is composed from ECG waveforms from two leads filtered using proprietary algorithm. From this time series heart rate (estimated in the periods of 5 seconds) and $\mathrm{R}-\mathrm{R}$ intervals are computed and enclosed into the data set. Additionally, breathing rate was estimated using elastic band and skin temperature are enclosed.

The main challenge for classification scenario in this particular data set is to generalize the same activities between different subjects. Certain physical activities can be performed in a very different way - this problem arises from number of reasons: starting from handedness, physical disposition, weight and height, etc. Those differences could be even so significant that they can be used for biometric authentication [8]. Moreover, sensor placement can be slightly different between session (although we put extra effort to ensure the same mounting places).

In Fig. 3 we depicted similarities of randomly selected $\sim 2 s$ sequences of data. To be more precise, we see there multidimensional scaling on the set of features of the data, i.e. solution to the following minimization problem

$$
\min _{x_{1}, \ldots, x_{N} \in \mathbb{R}^{2}} \sum_{1 \leq i<j \leq N}\left(\left\|x_{i}-x_{j}\right\|-\left\|f_{i}-f_{j}\right\|\right)^{2},
$$

where $f_{i} \in \mathbb{R}^{K}$ denotes feature vector of the $i$ th window. The feature vector is composed using statistics commonly used in solutions described in section III-D.

The bottom-right plot of Fig. 3 illustrates that the certain activity can be more similar to different activity than the same activity of different subject. Of course this strictly depends on classification model but, all in all, this shows that physical activity classification needs to be performed with respect to subject identification. Another problem is illustrated on top-right plot of the same figure. In the dataset there was left-handed subject and certain activities (for example throwing_hose) was performed in very distinctive way.

\section{AAIA'15 DATA Mining COMPETITION}

AAIA'15 Data Mining Competition took place between March 9, 2015 and June 5, 2015 at Knowledge Pit on-line platform. It was a continuation of the contest initiated during the previous edition of the data challenge associated with International Symposium on Advances in Artificial Intelligence and Applications (the AAIA conference series) [14]. This year's topic was related to real-time screening of firefighters' vital functions and monitoring of ongoing physical activities at the incident scene.

\section{A. The task description}

The objective in this competition was to devise efficient methods for automatic labelling of short series of the sensory data with basic activities of a firefighter. On the one hand, this task was very challenging due to a fact that different people tend to perform the same activities in different ways. On the other hand, automatically generated and accurate activity labels would facilitate monitoring of firefighters' safety and contribute to development of efficient command supporting systems [13], [14].

For the purpose of the competition we provided the acquired data in a tabular format as two separate sets. The training data set contained 20,000 rows and 17,242 columns. Each row corresponded to a short time series (approximately $1.8 s$ long) of sensory readings. Such a short time period was dictated by the applicability requirements and the available hardware setup (see Section II-A)

The first 42 columns represented aggregations of data from sensors monitoring firefighter's vital functions. The remaining columns were divided into 400 chunks that represented consecutive readings from the sets of kinetic sensors attached to firefighter's torso, hands, arms and legs (see the more detailed description in Section II). Therefore, a single chunk of columns consisted of 43 numeric values, from which the first one was time from the beginning of the series and the following 42 values represented the readings from the accelerometers (measured in $\mathrm{m} / \mathrm{s}^{2}$ ) and gyroscopes (measured in $\mathrm{deg} / \mathrm{s}$ ).

An average time difference between consecutive sensory readings in the data was $4.5 \mathrm{~ms}$. Labels for the training data were provided in a separate file. Each row in this file contained two labels for a corresponding row in the training data. The first label described a posture of a firefighter and the second described his current activity. In the original dataset the activity was described by 1 to 4 tags. For the competition we have provided only the main activity. Test data file was in the same format as the training data set, however, the labels for the test series were hidden from participants.

It is important to note that the training and test data sets consisted of recordings which were obtained from different groups of firefighters. Each of the sets contained the data acquired from only four persons and the scenario of the training exercises which they conducted was slightly different. There were no firefighter identifiers available in the data. By hiding this information from participants we wanted to promote 




Fig. 2. Accelerations from BSN. Three actions were selected and marked.

solutions which are insensitive to individual characteristics of particular firefighters and are able to cope with disturbances of the data. Those prerequisites were the main reason for the unbalanced distribution of decision labels and had a huge impact on the shape of the most successful solutions.

\section{B. Evaluation procedure}

The quality criterion in the competition was devised so that it reflected the specific requirements for the task. Since this task involved labelling sequences with tags that could take many different values with unbalanced representation in the data, the quality of submitted solutions was assessed using the balanced accuracy measure. This particular criterion had been already used in several others data mining competitions, e.g. [18]. It is insensitive to skewed distribution of decisions and thus promotes classifiers which are able to robustly identify labels of cases from minority classes.

The balanced accuracy (BAC) is typically defined as an average accuracy within all decision classes. In particular, if we denote a vector with predictions returned by a classifier by preds and a vector of true decision labels by labels, we may define BAC as:

$$
\begin{aligned}
& A C C_{i}(\text { preds }, \text { labels })=\frac{\mid\left\{j: \text { preds }_{j}=\text { labels }_{j}=i\right\} \mid}{\mid\left\{j: \text { labels } s_{j}=i\right\} \mid} \\
& B A C(\text { preds }, \text { labels })=\frac{1}{l} \sum_{i=1}^{l} A C C_{i}(\text { preds }, \text { labels })
\end{aligned}
$$

where $l$ is the total number of possible labels. In our compe- 
TABLE IV. THE FINAL AND PRELIMINARY RESULTS OF THE TOP-RANKED TEAMS.

\begin{tabular}{|l|c|c|c|}
\hline Rank & Team name & Preliminary & Final score \\
\hline 1 & jan & 0.85768 & 0.83912 \\
2 & zagorecki & 0.85184 & 0.82985 \\
3 & nitekna & 0.85015 & 0.8261 \\
4 & mathurin & 0.82523 & 0.80408 \\
5 & lp319499 & 0.80318 & 0.79137 \\
$\ldots$ & $\ldots$ & $\ldots$ & $\ldots$ \\
38 & baseline & 0.61414 & 0.60361 \\
\hline
\end{tabular}

tition, a value of BAC measure was separately computed for the two decision attributes (the posture and activity). Due to the fact that the decision attributes considerably differed in the number of possible values, we decided to assign them different weights which would compensate for the increased difficulty of predicting the activity label. As a result, the BAC values for the posture and activity attributes had a different impact on the evaluation score. If we denote the BAC value for the posture as $B A C p$ and the value for the activity as $B A C a$, we can determine the evaluation score of a solution $s$ as:

$$
\operatorname{score}(s)=\frac{B A C p(s)+2 \cdot B A C a(s)}{3} .
$$

The evaluation procedure in the competition was two-fold. During the course of the contest an on-line evaluation system was providing a constant feedback for the participants in a form of a publicly available leaderboard - a dynamic ranking of participant's best results. However, the scores displayed on the leaderboard were only a preliminary assessment of the solution's quality. They were computed using only $10 \%$ of available test data. After completion of the competition there were the second evaluation round. It was available only for those teams which had provided a description of their solution in a form of a short competition report. The final evaluation was carried out independently from the preliminary one, using the remaining part of the test data.

\section{Summary of the competition results}

AAIA'15 Data Mining Competition attracted skilled data mining practitioners from around the world. Comparing to the previous year's edition of the challenge there were noticeably more registered teams (152 in total, an increase by 36 teams), from which 79 actively participated in the challenge by submitting at least one solution (an increase by 22 teams). We received 1,840 correctly formatted solutions (an increase by $42 \%$ ) and the top-ranked participants have beaten the baseline solution by nearly 24 percentage points. Additionally, 50 teams provided a brief report describing their approach. Table IV shows scores obtained by the top-ranked teams.

The solutions submitted by participants proved to be a valuable source knowledge. They not only provided an insightful view on the state-of-the-art in multidimensional time series analysis but also contained inspiring new ideas, design specifically for the considered problem. The most interesting of these ideas are described by their authors in separate papers submitted for the competition track of AAIA'15 conference [2], [11], [15], [17], [19], [20].

\section{Summary of the most successful submissions}

The top-ranked participants of AAIA'15 Data Mining Competition managed to test effectiveness of a wide range of approaches to classification of high dimensional sensory data. However, nearly all of the successful solutions had a common denominator. They all utilized some sort of attribute engineering or feature extraction methods [12] in order to represent the time series data by a new set of attributes. In all cases, the main purpose of this data transformation was to define an attribute space which on the one hand was insensitive to differences in movement patterns between different firefighters and on the other hand, was characterized by a much lower dimensionality in comparison to the tabular representation of the competition data.

Among the new features used in the top solutions a large share corresponded to summary statistics, commonly used to describe sample distributions. These characteristics include typical location statistics (i.e. mean, centiles, time window minimum and maximum), shape statistics (i.e. skewness, kurtosis) and dispersion statistics (i.e. standard deviation, energy, range and difference between centiles). Several solutions also made use of characteristics measuring the dynamics of data, such as the mean, minimum and maximum difference between values of consecutive sensor readings. Finally, the two best solutions in the competition were using features derived from the Fast Fourier Transform of the data.

Having defined a suitable representation of data, the participants were employing standard machine learning algorithms to perform the classification. Among the most popular classifiers were the Random Forest [4] and Support Vector Machines [1]. The best results were obtained by teams which carefully tuned parameters of their learning algorithm. Interestingly, the topranked participants were using different approaches to tackle the problem of two decision attributes in the data. Some of the teams constructed two independent classifiers, whereas the others merged the two class labels into a single one and trained a single model for a multi-class prediction problem. However, the best performing classification model was firstly trained to predict the first label (the posture) and then, the obtained prediction was used as a new feature for prediction of the second label [15].

\section{CONCLUSION}

Physical activity classification based on inertial data from body sensor networks is a very challenging task. The main difficulty that needs to be addressed when solving this problem is the fact, that same activity can be performed in very different ways by different subjects. Moreover, due to severe hardware limitations, any classification scenario in a real-life application needs to find a trade-off between a classification accuracy and power efficiency. So far very little work has been done to address this issue and devise new methods that would be suitable for mobile, long-running platforms.

In this paper we described a data set that can be used in future studies on this important subject. We also summarized a data mining competition which we had organized in order to draw attention of data mining community and stimulate research in this type of application areas. In particular, solutions submitted by participants of the competition constitute 
a valuable insight regarding the state-of-the-art in the on-line activity recognition field.

\section{REFERENCES}

[1] B. E. Boser, I. M. Guyon, and V. N. Vapnik A training algorithm for optimal margin classifiers. In Proceedings of the Fifth Annual Workshop on Computational Learning Theory, COLT '92, pages 144-152, New York, NY, USA, 1992. ACM.

[2] M. Boull'e. Tagging Fireworkers Activities from Body Sensors under Distribution Drift. In M. Ganzha, L. A. Maciaszek, and M. Paprzycki, editors, Proceedings of FedCSIS 2015. IEEE, 2015.

[3] B. Brehmer. Strategies in Real-Time, Dynamic Decision Making. Insights in decision making, pages 262-279, 1990.

[4] L. Breiman. Random Forests. Machine Learning, 45(1):5-32, 2001.

[5] Department of Communities and Local Goverment. Fire Service Operations, Incident Command. Fire Service Manual. London TSO, third edition, 2008.

[6] Emergency Management Institute. Introduction to Incident Command System, ICS-100. http://training.fema.gov/EMIWeb/IS courseOverview.aspx?code=IS-100.b, 2013. Access: 22.02.201.

[7] R. Friedman, A. Kogan, and Y. Krivolapov. On power and throughput tradeoffs of wifi and bluetooth in smartphones. Mobile Computing, IEEE Transactions on, 12(7):1363-1376, July 2013.

[8] D. Gafurov, K. Helkala, and T. Søndrol. Biometric gait authentication using accelerometer sensor, 2006

[9] A. Graeger, U. Cimolino, H. de Vries, and J. Sümersen. Einsatzund Abschnittsleitung: Das Einsatz-Führungs-System (EFS). Ecomed Sicherheit, 2009.

[10] L. J. Grorud and D. Smith. The National Fire Fighter Near-Miss Reporting. Annual Report 2008. In An exclusive supplement to FireRescue magazine, pages 1-24. Elsevier Public Safety, 2008.

[11] M. Grzegorowski and S. Stawicki. Feature Extraction from Machine Generated Data. In M. Ganzha, L. A. Maciaszek, and M. Paprzycki, editors, Proceedings of FedCSIS 2015. IEEE, 2015.

[12] I. Guyon, S. Gunn, M. Nikravesh, and L. A. Zadeh, editors. Feature Extraction: Foundations and Applications. Studies in Fuzziness and Soft Computing. Springer, 2006.
[13] J. Huang, F. Qian, A. Gerber, Z. M. Mao, S. Sen, and O. Spatscheck. A close examination of performance and power characteristics of $4 \mathrm{~g}$ lte networks. In Proceedings of the 10th International Conference on Mobile Systems, Applications, and Services, MobiSys '12, pages 225238, New York, NY, USA, 2012. ACM.

[14] A. Janusz, A. Krasuski, S. Stawicki, M. Rosiak, D. Ślęezak, and H. S. Nguyen. Key Risk Factors for Polish State Fire Service: a Data Mining Competition at Knowledge Pit. In M. Ganzha, L. A. Maciaszek, and M. Paprzycki, editors, Proceedings of FedCSIS 2014, pages 345-354. IEEE, 2014.

[15] J. Lasek and M. Gagolewski. The Winning Solution to the AAIA?15 Data Mining Competition: Tagging Firefighter Activities at a Fire Scene. In M. Ganzha, L. A. Maciaszek, and M. Paprzycki, editors, Proceedings of FedCSIS 2015. IEEE, 2015.

[16] M. Meina, B. Celmer, and K. Rykaczewski. Towards robust framework for on-line human activity reporting using accelerometer readings. In D. Ślęzak, G. Schaefer, S. Vuong, and Y.-S. Kim, editors, Active Media Technology, volume 8610 of Lecture Notes in Computer Science, pages 347-358. Springer International Publishing, 2014.

[17] S. Wawrzyniak and W. Niemiro. Clustering Approach to the Problem of Human Activity Recognition using Motion Data. In M. Ganzha, L. A. Maciaszek, and M. Paprzycki, editors, Proceedings of FedCSIS 2015. IEEE, 2015.

[18] M. Wojnarski, A. Janusz, H. S. Nguyen, J. Bazan, C. Luo, Z. Chen, F. Hu, G. Wang, L. Guan, H. Luo, J. Gao, Y. Shen, V. Nikulin, T.-H. Huang, G. J. McLachlan, M. Bošnjak, and D. Gamberger. RSCTC'2010 discovery challenge: Mining DNA microarray data for medical diagnosis and treatment. In M. S. Szczuka et al., editor, Proceedings of RSCTC'2010, volume 6086 of LNAI, pages 4-19, Heidelberg, 2010.Springer.

[19] A. Zagorecki. A Versatile Approach to Classification of Multivariate Time Series Data. In M. Ganzha, L. A. Maciaszek, and M. Paprzycki, editors, Proceedings of FedCSIS 2015. IEEE, 2015.

[20] E. Zdravevski, P. Lameskiy, R. Mingov, A. Kulakov, and D. Gjorgjevikj. Robust histogram-based feature engineering of time series data. In M. Ganzha, L. A. Maciaszek, and M. Paprzycki, editors, Proceedings of FedCSIS 2015. IEEE, 2015. 